\title{
INVENTARIO DE CUMALAS (MYRISTICACEAE) EN LA AMAZONÍA PERUANA
}

\author{
Ricardo ZÁRATE ${ }^{1}$, Tony J. MORI ${ }^{2}$, Luis A. VALLES ${ }^{1}$, José T. MACO ${ }^{1}$ \\ 1 Instituto de Investigaciones de la Amazonía Peruana. Programa de Investigación en Cambio Climático Desarrollo \\ Territorial y Ambiente (Proterra); Av. Quiñones km 2.5, San Juan Bautista, Maynas, Loreto, Perú. E-mails: \\ rzarate@iiap.org.pe, ric_zarate@hotmail.com \\ 2. Servicios de Biodiversidad; Jr. Independencia 405, Punchana, Maynas, Loreto, Perú. E-mail; \\ tmori@serviciosdebiodiversidad.com
}

\section{RESUMEN}

Evaluamos la diversidad, composición y estructura de Myristicaceae $\geq 10 \mathrm{~cm}$ de DAP (Diámetro a la Altura del Pecho, a $1.3 \mathrm{~m}$ del suelo) en 100 parcelas rectangulares, 76 de 50 × 20 y 24 de 50 x $10 \mathrm{~m}$ ( 8.8 ha en total $)$ en la Amazonía peruana (departamentos de Loreto, Huánuco, Junín, Madre de Dios, Ucayali, Ayacucho, Cusco y Junín). Reportamos 390 árboles $\geq 10 \mathrm{~cm}$ de DAP, correspondiendo a 44 especies y 5 géneros. La diversidad alfa varió de 0 a 14.49 de acuerdo a $\alpha$ Fisher, de 0 a 1.972 de acuerdo a Shannon $\mathrm{H}$ y de 0 a 0.843 de acuerdo a Simpson 1-D. La diversidad beta fue baja con menos del $25 \%$ de similitud florística entre las parcelas. En cuanto a la riqueza de especies tenemos: Virola: 23 especies (52.3\%), Iryanthera: 16 especies (36.4\%). Las especies que incluyen la mayor cantidad de individuos son: Virola pavonis, Iryanthera laevis, Otoba glycycarpa, Virola calophylla y Otoba parvifolia; las cuales incluyeron el $52 \%$ del total de individuos. Los géneros que presentaron la mayor cantidad de individuos fueron: Virola e Iryanthera alcanzando el $82 \%$ del total de individuos. La mayor riqueza y abundancia de especies la reportamos en el norte y centro de la Amazonía peruana. El promedio de tallos fue de 44.3 ind. ha ${ }^{-1}$. Solamente 66 parcelas presentaron $\geq 1$ individuo de Myristicaceae, alcanzando 6.1 ha (69.3\%), con densidades de 1 a 6 individuos en parcelas de 0.05 ha y de 1 a 23 en parcelas de 0.1 ha. La diversidad, composición y estructura de Myristicaceae fue variable en la Amazonía peruana.

PALABRAS CLAVE: Myristicaceae, Virola, Iryanthera, Compsoneura, Osteophloeum, Otoba, Composición, Diversidad.

\section{INVENTORY OF “CUMALAS” (MYRISTICACEAE) IN THE PERUVIAN AMAZON}

\begin{abstract}
We evaluated the diversity, composition and structure within Myristicaceae $\geq 10 \mathrm{~cm} \mathrm{DBH}$ (diameter at $1.3 \mathrm{~m}$ above the ground) in 100 rectangular plots, 76 of $50 \times 20$ and 24 of $50 \times 10 \mathrm{~m}$ (8.8 ha in total) in the Peruvian Amazon (departments of Loreto, Huanuco, Junin, Madre de Dios, Ucayali, Ayacucho, Cusco and Junín). We reported 390 trees $\geq 10 \mathrm{~cm} \mathrm{DBH}$, corresponding to 44 species and 5 genera. Alpha diversity varied from 0 to 14.49 according to $\alpha$ Fisher, from 0 to 1.972 according to Shannon $\mathrm{H}$ and from 0 to 0.843 according to Simpson 1-D. Beta diversity was low with less than $25 \%$ of floristic similarity between plots. In terms of species richness, we have Virola: 23 species (52.3\%), Iryanthera: 16 species $(36.4 \%)$. The species that include more individuals were: Virola pavonis, Iryanthera laevis, Otoba glycycarpa, Virola calophylla and Otoba parvifolia, which include $52 \%$ of all individuals. The genera that had the highest number of individuals were: Virola and Iryanthera reaching a $82 \%$ of all individuals. The greatest richness and abundance of the species were reported at the north and center of the peruvian Amazon. Stems average was 44.3 ind stems ha1. Only 66 plots showed at least one individual of Myristicaceae, reaching 6.1 ha (69.3\%), at densities of 1 to 6 individuals in 0.05 ha plots and 1 to 23 in 0.1 ha plots. Diversity, composition and structure varies within Myristicaceae in the peruavian Amazon.
\end{abstract}

KEYWORDS: Myristicaceae, Virola, Iryanthera, Compsoneura, Osteophloeum, Otoba, Composition, Diversity. 


\section{INTRODUCCIÓN}

La familia Myristicaceae fue establecida por Robert Brown hace más de doscientos años (1810). La familia es conocida mundialmente por la nuez moscada (Myrstica fragans), una especie cuyo origen es de Indonesia y tiene valor comercial.

Actualmente se ha reportado aproximadamente 500 especies incluidas en 18-21 géneros a nivel global (Ribeiro et al., 1999; Janovec, 2000; y Stevens, 2001 onwards), de los cuales 83 especies en 5 géneros fueron reportados para el Neotropico (Ribeiro et al., 1999); y en el Perú se ha reportado: 39 especies en 5 géneros (Brako \& Zarucchi, 1993; Janovec, 2002; Vásquez et al., 2002; Vásquez \& Rojas, 2004). En la Amazonía peruana esta familia está representada principalmente por árboles (raro arbustos), dióicos (algunos monoicos), con hojas simples alternas, exudados que oxidan en colores translúcidos a rojizo o amarillento, con olores fuertes en la corteza interna, inflorescencias en fascículos o racimos, cimas, cabezuelas o panículas de fascículos; flores unisexuales, actinomorfas, apétalas, perianto gamotépalo, ovario súpero; frutos drupa dehiscente o cápsula (Warburg, 1897; Smith 1937; Cronquist, 1988; Spichiger et al., 1989; Gentry, 1993; Vásquez, 1997; Ribeiro et al., 1999; Ayala, 2003; Amasifuen \& Zárate, 2005).

La importancia ecológica de la familia radica en que varias de sus especies son abundantes en los bosques de la Amazonía peruana, y esta generalmente reportada entre las diez familias más abundantes (Terborgh \& Andresen, 1998; Haugaasen \& Peres, 2006; Wittmann et al., 2006; Pitman et al., 2008). También son micro-hábitat de diversos organismos vivos, desde aves, reptiles, insectos y hasta arácnidos, quienes encuentran en la arquitectura peculiar de este grupo de árboles un lugar especial donde habitar. Por otra parte, muchas de las especies de la familia Myristicaceae son usadas comúnmente por los pobladores locales como madera de aserrío, para la construcción de viviendas rurales y demás construcciones. También es muy requerida por la industria maderera a mayor escala, quienes años tras otro vienen extrayendo considerables volúmenes de especímenes de "cumala".

Este trabajo contribuirá con información básica de la diversidad, composición y estructura de los árboles y arbustos de Myristicaceae con la finalidad de generar información para el manejo adecuado de estas especies. Ya que la creciente deforestación y la presión del uso maderable de estas especies pone en peligro el equilibrio ecológico intra e inter poblacional de las mismas.

El presente trabajo tiene el objetivo de ejecutar inventarios de los individuos de Myristicaceae en la Amazonía peruana para la evaluación de la diversidad, composición y estructura.

\section{MATERIAL Y MÉTODOS}

\section{ÁREA DE ESTUDIO}

El muestreo se ejecutó en la Amazonía peruana, en los departamentos de Loreto, Huánuco, Junín, Madre de Dios, Ucayali, Ayacucho, Cusco y Junín (Tabla 1, Figura 1). De acuerdo a Josse et al. (2007) los nuestros se ejecutaron en las siguientes Sistemas Ecológicos: Bosque con bambú del suroeste de la Amazonía, Bosque inundable de la llanura aluvial de ríos de aguas blancas del oeste de la Amazonía, Bosque inundable y vegetación riparia de aguas negras del oeste de la Amazonía, Bosque pantanoso de la llanura aluvial del oeste de la Amazonía, Bosque pantanoso de palmas de la llanura aluvial del oeste de la Amazonía, Bosque siempreverde de la penillanura del oeste de la Amazonía, Bosque siempreverde estacional de la penillanura del suroeste de la Amazonía, Bosque siempreverde subandino del suroeste de la Amazonía, Bosques siempreverde de la penillanura del Oeste de la Amazonía, Bosques siempreverde subandino del oeste de la Amazonía, Bosques siempreverde subandino del suroeste de la Amazonía, y Complejo de bosques sucesionales inundables de aguas blancas de la Amazonía

\section{PARCELAS Y ORGANISMOS MUESTREADOS}

Se establecieron 100 parcelas rectangulares, 76 de 50 x 20 (que corresponde a las parcelas Whittaker modificadas (Stohlgren et al., 1995)) y 24 de 50 x 10 $\mathrm{m}$. Los datos que se registraron de las parcelas fueron: ubicación, altitud, comunidad vegetal, localidad. Dentro de este inventario se ha muestreado los individuos de árboles (o arbustos) de la familia Myristicaceae mayores o iguales a $10 \mathrm{~cm}$ de diámetro a la altura de $1.3 \mathrm{~m}$ sobre el suelo.

\section{HERBORIZACIÓN}

Recolecta.- Las colectas botánicas se realizaron con tijeras podadoras de mano y telescópicas, subidores de árboles, prismáticos, costales, bolsas plásticas y otros. Codificación.- Todas las colectas fueron rotuladas con códigos numéricos. Registro.- De cada individuos se ha registrado la siguiente información: nombre preliminar de la especie, altura total, diámetro a $1.3 \mathrm{~m}$ de altura sobre el suelo, presencia de flores y o frutos, olor de la corteza, color y abundancia de "látex" y código de parcela; esta información es útil para la identificación de las especies. Prensado.- El prensado ha iniciado con la inclusión de las colecciones en camisetas de papel periódico, finalmente, este proceso termino con el prensado en el secador. Preservado.- El preservado de las colecciones se realizo con alcohol al 75\% colocadas dentro de bolsas plásticas gruesas.

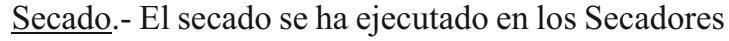


del IIAP, AMAZ, USM y Servicios de Biodiversidad. Identificación.- Las muestras se identificaron desde el momento de la colecta, luego se prosiguió revisando las claves de: Warburg, 1897; Smith, 1937; Cronquist, 1988; Spichiger et al., 1989; Gentry, 1993; Vásquez, 1997; Ribeiro et al., 1999; Ayala, 2003; Vásquez \& Rojas, 2004; Amasifuen \& Zárate, 2005; y comparando con las exicatas de los Herbarios HH, AMAZ y USM. Depósito.- Las colectas se depositaron en el Herbario Herrerense (HH) del Instituto de Investigaciones de la Amazonía Peruana-IIAP.

\section{ANÁLISIS DE DATOS}

Se ha calculado la Diversidad alfa (ShanomWiver y AlfaFisher) y beta (Simpson y Bray-Curtis).

Calculamos el número y la abundancia de las especies de Myristicaceae, indicando el porcentaje de cada valor respecto al número total de especies e individuos encontrados en cada parcela. Para cada especie de Myristicaceae, calculamos la abundancia (porcentaje de individuos del total de individuos de Myristicaceae). Estos valores fueron utilizados para representar la importancia ecológica de cada especie. De manera espacial, representamos estos porcentajes con el fin de detectar áreas de mayor riqueza y abundancia de especies de Myristicaceae en la Amazonía peruana. Para la determinación de las clases diamétricas, ancho de las clases y número de clases se utilizó las distribuciones de frecuencia, con las siguientes fórmulas: rango $(\mathrm{R})=$ (valor mayor - valor menor; cantidad de clases $(\mathrm{K})=1+$ 3,322 $\log (\mathrm{N})$; y la amplitud de la clase $(\mathrm{C}): \mathrm{C}=\mathrm{R} / \mathrm{K}$ (Orozco \& Brumer, 2002).

\section{RESULTADOS Y DISCUSIÓN}

\section{DIVERSIDAD}

Diversidad alfa. La diversidad alfa fue baja varia de 0 hasta 14.49 de acuerdo a $\alpha$ Fisher, de 0 a 1.972 de acuerdo a Shannon H y de 0 a 0.843 de acuerdo a Simpson 1-D. De acuerdo a Simpson 1-D las clases de diversidad alfa más frecuentes fueron $0.6 \leq 0.7 \mathrm{y}$ $0.7 \leq 0.8$ en la que se agrupa el $43.9 \%$, y otra clase importante es $0 \leq 0.3$ que presenta el $24 \%$ de las parcelas. Asimismo, de acuerdo al índice de $\alpha$ Fisher las clase que mayor unidades de muestreo contiene es $0 \leq 2$ la cual contiene más del $50 \%$ del total de parcelas (Tabla 1). Mientras que Spichiger et al., 1989; Acevedo et al., 1997; Pitman et al., 1999, 2001a, 2001b, 2003; Vriesendorp et al., 2004; Ríos \& Dávila, 2005; Vásquez \& Rojas, 2005; Valderrama, 2007; encontraron resultados similares en cuanto a $\alpha$ Fisher (de 0.20 a 12.01), y ciertas diferencias en Shannon H (de 0 a 2.626) y Simpson 1-D (de 0 a 0.909) para parcelas de 1 ha en la Amazonía peruana; estas diferencias se explican por: i. la Amazonía peruana varía dramáticamente en diversidad y composición florística de lugar en lugar (Gentry \& Ortiz, 1993) con diversidad de hábitats y microhábitats (Leigh, 1986), influenciado, entre otras fuerzas, por: la menor precipitación y mayor estacionalidad de la precipitación hacia el sur (Kalliola \& Puhakka, 1993); suelos altamente heterogéneos (Linna, 2003; Escobedo, 2007a, 2012a); fisiografía diversa (Escobedo, 2007b, 2012 b), con varias comunidades vegetales (Encarnación, 1985, 1993; IIAP-BIODAMAZ, 2004; Josse et al., 2007). Y ii. el tamaño del área muestreada. Además la baja diversidad de la familia se explica a que solamente se está considerando el taxón familia y a que varias de sus especies son nítidamente abundantes.

Diversidad Beta. La similitud entre las parcelas varió desde cero hasta uno de acuerdo a los índices: Jaccard, Simpson y Bray-Curtis con promedios de $0.091,0.225$ y 0.101 , respectivamente; indicando una baja similitud entre las parcelas establecidas; contrariamente a lo reportado por Spichiger et al., 1989; Acevedo et al., 1997; Pitman et al., 1999, 2001a, 2001b, 2003; Vriesendorp et al., 2004; Ríos \& Dávila, 2005; Vásquez \& Rojas, 2005; Valderrama, 2007; en la que encontraron mediana similitud, lo cual debe estar relacionado al tamaño de la parcela y a la homogeneidad de comunidades vegetales. De acuerdo al índice cualitativo de similaridad de Simpson de la composición florística de las parcelas muestreadas se forman al menos 7 grupos, las cuales tienden a agruparse en tres grandes comunidades vegetales que podríamos llamarlos de la siguiente manera: 1.- Bosques inundables o inundados; 2. Bosques de colinas bajas y 3.- Bosques de montañas, colinas y planicies no inundables. Sin embargo, hay varias parcelas que pertenecen a una de las grandes comunidades vegetales y por el agrupamiento de la similitud florística están ubicadas en otras grandes comunidades vegetales (Figura 2); lo cual es concordante con el análisis de Spichiger et al., 1989; Acevedo et al., 1997; Pitman et al., 1999, 2001a, 2001b, 2003; Vriesendorp et al., 2004; Ríos \& Dávila, 2005; Vásquez \& Rojas, 2005; Valderrama, 2007. Así mismo de acuerdo a los índices de similitud cuantitativa de Bray-Curtis las cumalas forman nueve grupos en las cuales se define más claramente la similitud entre las parcelas en los bosques de colinas bajas, mientras tienen a traslaparse los bosques de montañas, colinas altas, planicies no inundables e inundables (Figura 3). 


\section{COMPOSICIÓN FLORÍSTICA}

Se reporta 390 tallos $\geq 10 \mathrm{~cm}$ DAP, incluidas en 44 especies y 5 géneros. La familia Myristicaceae presenta 40 especies reportadas para el Perú (Brako \& Zarucchi, 1993; Janovec, 2002; Vásquez et al., 2002;) y en este trabajo reportamos 32 especies con nombres latinos completos, las especies que no reportamos son: Compsoneura diazii, Iryanthera coriacea, Iryanthera longiflora, Iryanthera paradoxa, Iryanthera polyneura, Virola caducifolia, Virola marlenei y Virola weberbaueri, probablemente por su distribución espacial, preferencias de hábitats y microhábitats, abundancia baja de individuos o por tender a tener menos de 10 $\mathrm{cm}$ de DAP en estado adulto. Reportamos 12 morfoespecies los cuales pueden ser nuevos reportes para el país o nuevas especies para la ciencia. Especialmente, los géneros Iryanthera y Virola cuentan con una gran variedad de especies y variación morfológica dificultando la determinación. Más aún existen muchos morfotipos intermedios, indicando que probablemente algunas de estas especies podrían generar híbridos entre ellas (p.e. Virola sebifera y V. loretensis (Steeves 2011).

Las diez especies que incluyen la mayor cantidad de individuos en nuestro estudio son: Virola pavonis (21.1\%), Iryanthera laevis (11\%), Otoba glycycarpa (9\%), Virola calophylla (7.2\%), Otoba parvifolia (4.9\%), Iryanthera juruensis (4.4\%), Iryanthera paraensis (4.4\%), Iryanthera tricornis (4.1\%), Iryanthera hostmannii (3.6\%), e Iryanthera tessmannii (3.3\%); lo cual concuerda con lo publicado por Spichiger et al., 1989; Acevedo et al., 1997; Pitman et al., 1999, 2001a, 2001b, 2003; Vriesendorp et al., 2004; Ríos \& Dávila, 2005; Vásquez \& Rojas, 2005; excepto por cuatro especies, las cuales son: Virola sebifera, Iryanthera elliptica, Osteophloeum platyspermum e Iryanthera olacoides. Los géneros que presentaron la mayor cantidad de individuos en nuestros reportes fueron: Virola e Iryanthera alcanzando el $82 \%$ del total de individuos, igualmente a lo reportado por Spichiger et al., 1989; Acevedo et al., 1997; Pitman et al., 1999, 2001a, 2001b, 2003; Vriesendorp et al., 2004; Ríos \& Dávila, 2005; Vásquez \& Rojas, 2005; en la cual los géneros Virola e Iryanthera alcanzaron el $84 \%$. En cuanto a la riqueza de especies tenemos: Virola: 23 especies (52.3\%), Iryanthera: 16 especies (36.4\%), Compsoneura: 2 especies (4.5\%), Otoba: 2 especies (4.5\%) y Osteophloeum: 1 especie (2.3\%), ver Tabla 3 para mayores detalles. Estas diferencias se explican por la heterogeniedad microhábitats y por la forma y tamaño del área muestreada.

Localidades al norte (departamento de Loreto) y centro (departamentos de Huánuco y Junín) del Perú contienen la mayor riqueza y densidad en especies de Myristicaceae (Figura 4a y 4b), que las localidades más al sur (departamento de Madre de Dios). Este patrón es similar a la distribución espacial de diversidad arbórea en el Perú, con valores de diversidad alta en el norte y menores valores en el sur (Ter Steege et al., 2003). La alta riqueza de especies podría estar relacionado a la gran variación edáfica y geológica encontrada en el norte del país, que genera también una alta variación en las tasas de recambio de especies (Pitman et al., 2008; Kalliola \& Puhakka, 1993).

\section{ESTRUCTURA FISONÓMICA}

En el presente trabajo el 69.3\% del área muestreada (6.1 ha) presenta al menos 1 individuo de Myristicaceae, teniendo en cuenta estas parcelas, reportamos en promedio 44.3 ind. $\geq 10 \mathrm{~cm}$ de DAP ha $^{-1}$, en nuestras parcelas, con una variación de 1 a 6 individuos en las parcelas de 50 x $10 \mathrm{~m}$ y de 1 a 23 en las parcelas de 50 × $20 \mathrm{~m}$; mientras que Spichiger et al., 1989; Acevedo et al., 1997; Pitman et al., 1999, 2001a, 2001b, 2003; Vriesendorp et al., 2004; Ríos \& Dávila, 2005; Vásquez \& Rojas, 2005; encontraron el $93.6 \%$ de las parcelas evaluadas con Myristicaceae, en promedio con 29.1 ind. ha ${ }^{-1}$ en 40 parcelas de 1 ha (2.91 ind. $0.1 \mathrm{ha}^{-1}$ ), con una variación de 6 a 94 individuos en parcelas de $100 \mathrm{x}$ $100 \mathrm{~m}$, es decir de 0.6 a 9.4 ind. $0.1 \mathrm{ha}^{-1}$; estas diferencias se explican por el tamaño de las muestras y por la variación de las comunidades vegetales. El análisis de las clases de DAP indican que se ajustan a una " $J$ " invertida, las clases con mayor cantidad de individuos son: $10 \leq 23 \mathrm{~cm}$ y $23 \leq 36 \mathrm{~cm}$ con 246 ind. $(63.1 \%)$ y 80 ind. (20.5\%) respectivamente, incluyendo más del $80 \%$ del total de tallos; mientras que las clases: $114 \leq 127 \mathrm{~cm}$ y $127 \leq 140 \mathrm{~cm}$ alcanzaron a tener un individuo (Tabla 4 y Figura 4). Las especies que presenta los mayores DAP son: Virola pavonis (hasta $140 \mathrm{~cm}$ ), Otoba parvifolia (hasta $115 \mathrm{~cm}$ ), Virola flexuosa (hasta $90 \mathrm{~cm}$ ), Otoba glycycarpa (hasta $89 \mathrm{~cm}$ ), Virola multiflora (hasta 80 $\mathrm{cm}$ ), Osteophloeum platyspermum (hasta $78 \mathrm{~cm}$ ) y otras (lo cual concuerda con lo publicado por Freitas, 1996; Valderrama, 2007; contrariamente las de menores DAP son: Virola calophylla, Iryanthera laevis, Iryanthera crassifolia, Iryanthera tessmannii, Virola loretensis, Virola mollissima, Iryanthera paraensis, Virola elongata, e Iryanthera lancifolia. El área basal total para los 8.8 ha muestreadas fue de $31.345734 \mathrm{~m}^{2}$, las especies con la mayor cantidad de área basal son: Virola pavonis (11.4948 m², 36.67\%), Otoba glycycarpa (4.3227 $\left.\mathrm{m}^{2}, 13.79 \%\right)$, Otoba parvifolia $\left(3.7163 \mathrm{~m}^{2}, 11.86 \%\right)$, Iryanthera laevis $\left(1.6474 \mathrm{~m}^{2}, 5.26 \%\right)$, Osteophloeum platyspermum $\left(1.2885 \mathrm{~m}^{2}, 4.11 \%\right)$, Virola flexuosa (1.0307 $\left.\mathrm{m}^{2}, 3.29 \%\right)$, Iryanthera 
hostmannii $\left(0.7816 \mathrm{~m}^{2}, 2.49 \%\right)$, juntas suman un poco más del $75 \%$ del total; lo cual concuerda con Freitas (1996), ya que estas especies tienden a presentar varios individuos y a la vez fustes anchos.

\section{AGRADECIMIENTOS}

A NatureServe, Gobierno Regional de Madre de Dios, Gobierno Regional de Loreto, Devida, quienes co-financiaron parte de este proyecto. A los pobladores locales, a las autoridades de gobiernos locales, provinciales y regionales por permitirnos el acceso a los muestreos en sus territorios. A los Herbarios Herrerense, AMAZ y USM por las facilidades brindadas en el uso de sus instalaciones. A Nicole Mitidieri por su apoyo durante la fase de campo. A Hamilton Beltran, Severo Baldeon, Marcos Ríos, Fredy Ramírez, Luis Torres, Carlos Mogollon, Jeancarlo Padilla, por su contribución en la identificación de las especies. Manuel Ahuite, Dillys Vela, Elvis Valderrama, Miguel Pacheco, Karla Meza, Nelly Maquera, Luis Mancilla, Clelia Rengifo, Joanela Reyna, Diego Macedo, Daniel Escobedo, por su asistencia en la colecta de información en campo. A Lizardo Fachín, Juan Palacios y Marcial Martínez por su apoyo en el manejo del sistema de información geográfica.

\section{BIBLIOGRAFÍA CITADA}

Acevedo, P.; Bell, D.; Rankin, K.; Smith, S. 1997. Floristric Composition, Structure, and Diversity Assessment in the Lower Urabamba Region. In: Alonso, A.; Dallmeieri, F.; Mistry, S.; Ros, C., Comiskey, J. Biodiversity Assessment in the Lower Urabamba Region. p. 45-58.

Amasifuen, C; Zárate, R. 2005. Composición Taxonómica, Ecología y Periodo de Floración de Plantas Leñosas "Dicotiledóneas". Tesis de pre-grado, Universidad Nacional de la Amazonía Peruana, Facultad de Ciencias Biológicas, Iquitos, departamento de Loreto, Perú. 397pp.

Ayala, F. 2003. Taxonomía Vegetal: Gymnospermae y Angiospermae de la Amazonía Peruana. Vol. 1 y 2. Iquitos. Primera Edición. Edit. CETA. 85pp.

Brako, L.; Zarucchi, J. 1993. Catalogue of thefloweringplants and gymnosperms of Peru [Catálogo de las angiospermas y gimnospermas del Perú]. Monogr. Syst. Bot. Missouri Bot. Gard. 45: 1286 pp.

Cronquist, A. 1988. Outline of Classification of Magnoliophyta. The Evolution and Classification of Flowering Plants. The New York Botanical Garden. Brox. New York. USA. 516pp.
Encarnación, F. 1985. Introducción a la flora y vegetación de la Amazonía Peruana: estado actual de los estudios, medio natural y ensayo de claves de determinación de las formaciones vegetales de la llanura Amazónica. Candollea, 40(1):237-252.

Encarnación, F. 1993. El bosque y las formaciones vegetales en la llanura amazónica del Perú. Alma Mater, 6: 93-114.

Escobedo, R. 2007a. Suelos y Capacidad de uso mayor de las tierras. Proyecto Microzonificación Ecológica y Económica del departamento de San Martín. Instituto de Investigaciones de la Amazonía Peruana. Iquitos. 172pp.

Escobedo, R. 2007b. Fisiografía. Proyecto Microzonificación Ecológica y Económica del departamento de San Martín. Instituto de Investigaciones de la Amazonía Peruana. Iquitos. $51 \mathrm{pp}$.

Escobedo, R. 2012a. Suelos y Capacidad de uso mayor de las tierras. Proyecto Microzonificación Ecológica y Económica de la carretera Iquitos-Nauta. Instituto de Investigaciones de la Amazonía Peruana. Iquitos. $115 \mathrm{pp}$.

Escobedo, R. 2012b. Fisiografía. Proyecto Microzonificación Ecológica y Económica de la carretera Iquitos-Nauta. Instituto de Investigaciones de la Amazonía Peruana. Iquitos. $35 \mathrm{pp}$.

Freitas, L. 1996. Caracterización Florística y Estructural de cuatro comunidades boscosas de Terraza baja en la zona de Jenaro Herrera, Amazonía Peruana. Instituto de Investigaciones de la Amazonía Peruana. Iquitos. 77pp.

Gentry, A. 1993. A Field Guide to the Families and Genera of Woody Plants of Northwest South America (Colombia, Ecuador, Perú) with supplementary notes on herbaceous taxa. Conservation International. Washington. 895pp.

Gentry, A.H.; Ortiz, R. 1993. Patrones de Composición Florística en la Amazonía Peruana. Amazonía Peruana. In Kalliolla, R; Puhakka, M. y Danjoy, W. (Eds) Vegetación húmeda tropical en el llano subandino. p. 155166.

Haugaasen, T.; Peres, C. 2006. Floristic, edaphic and structural characteristics offlooded and unflooded forests in the lower Rio Purús region of central Amazonia, Brazil. Acta Amazonica 36(1): 25-36.

Janovec, J.P. 2000. A systematic study of Compsoneura (A. DC.) Warb., A Neotropical member of the nutmeg family. Tesis de postgrado Texas A\&M University Estados Unidos: $359 \mathrm{pp}$. 
Janovec, J.P. 2002. Compsoneura camilodiazii J. Janovec, an interesting new species from the Rio Cenepa area, Peru. Novon 12(3):366-369.

Josse, C., G. Navarro, F. Encarnación, A. Tovar, P. Comer, W. Ferreira, F. Rodríguez, J. Saito, J. Sanjurjo, J. Dyson, E. Rubin de Celis, R. Zárate, J. Chang, M. Ahuite, C. Vargas, F. Paredes, W. Castro, J. Maco y F. Reátegui. 2007. Sistemas Ecológicos de la Cuenca Amazónica de Perú y Bolivia. Clasificación y mapeo. NatureServe, Virginia. 94 pp.

Kalliola, R.; Puhakka, M. 1993. Geografía de la selva baja peruana; In Kalliolla, R; Puhakka, M.; Danjoy, W. (Eds) Vegetación húmeda tropical en el llano subandino. p. 9-21.

IIAP-BIODAMAZ. 2004. Diversidad de Vegetación de la Amazonía peruana expresada en un mosaico de imágenes de satélite. Instituto de Investigaciones de la Amazonía Peruana, Iquitos, $74 \mathrm{pp}$.

Leigh, E. 1986. ¿Por qué hay tantos tipos de árboles tropicales?. In Rand, A., Windsor, D y Leigh, E. (Eds). Ecología de un bosque tropical: ciclos estacionales y cambios a largo plazo. p. 75-94.

Linna, A. 2003. Factores que contribuyen a las características del sedimento superficial en la selva baja de la Amazonía peruana. In Kalliolla, R; Puhakka, M. y Danjoy, W. (Eds) Vegetación húmeda tropical en el llano subandino. p. 87-97.

Orozco, L.; Brumer, C. 2002. Inventarios Forestales para Bosques Latifoliados de América Central. CATIE. Serie Técnica. Manual Técnico/CATIE $\mathrm{N}^{\circ}$ 50. Turrialba, Costa Rica. $264 \mathrm{pp}$.

Pitman, N.; Beltran, H.; Foster, R.; García, R.; Vriesendorp, C.; Ahuite, M. 2003. Flora y Vegetación. In: Pitman, N.; Vriesendorp, C.; Moskovits, D. (eds.). Peru: Yavarí Rapid Biological Inventories Report 11. Chicago: The field Museum. P. 52-59.

Pitman, N.; Mogollón, H.; Dávila, N.; Ríos, García, R.; Guevara, J.; Baker, T.; Monteagudo, A.; Phillips, O.; Vásquez, R.; Ahuite, M.; Aulestia, M.; Cardenas, D.; Cerón, C.; Loizeau, P.; Neill, D.; Núñez, P.; Palacios, W.; Spichiger, R.; Valderrama, E. 2008. Tree Community Change across $700 \mathrm{~km}$ of Lowland Amazonian Forest from the Andean Foothills to Brazil. BIOTROPICA $10.1111 / \mathrm{j} .1744$ 7429.2008.00424x. 1-11.

Pitman, N.; Terborgh, J.; Núñez. P.; Silman, M. 2001a. Especies arbóreas comunes de la parte baja de Madre de Dios, Perú. In: Rodríguez, L. O. (ed.), El Manu y otras experiencias de investigación y manejo de bosques neotropicales. Proyecto Aprovechamiento y Manejo Sostenible de la Reserva de Biosfera y Parque Nacional del Manu (Pro-Manu). p. 4652.
Pitman, N.; Terborgh, J.; Silman, M.; Nuñez, P.; Neill, D.; Cerón, C.; Palacios, W.; Aulestia, M. 2001. Dominante and Distribution of Tree Species in Upper Amazonian Terra Firme Forests. Ecology, 82 (8). 2101-2117.

Ribeiro, J.; Hopkins, M.; Vicentini, A.; Sothers, C.; Costa, M.; Brito, J.; Souza, M.; Martins, L.; Lohmann, L.; Assuncao, P.; Pereira, E.; Silva, C.; Mesquita, M.; Procopio, L. 1999. Flora da Reserva Ducke. Guia de identificaçao das plantas vasculares de uma floresta de terra-firme na Amazônia Central. Midas Printing. INPA. Manaus. 799pp.

Ríos, M; Dávila, N. 2005. Composición Florística, estructura y Diversidad de un Bosque de Tierra Firme en la Estación Biológiva Quebrada Blanco, Río Tahuayo, Loreto-Perú. Tesis de pregrado, Universidad Nacional de la Amazonía Peruana, Facultad de Ciencias Biológicas, Iquitos. Departamento de Loreto, Perú. 65pp.

Smith, A. C. 1937. The American species of Myristicaceae. Brittonia 2(5): 393-510.

Spichiger, R.; Méroz, J; Loizeau, P.; L. Stutz. 1989. Contribución a la Flora de la Amazonía Peruana: Los Árboles del Arboretum Jenaro Herrera. Vol. I 359pp. y Vol. II. 565pp.

Steeves, R. 2011. An Intrageneric and Intraspecific Study of Morphological and Genetic Variation in the Neotropical Compsoneura and Virola (Myristicaceae). Tesis de post-grado, The University of Guelph. Canada. 308pp.

Stevens, P. F. (2001 onwards). Angiosperm Phylogeny Website. Version 12, July 2012 [and more or less continuously updated since]." will do. (http://www.mobot.org/MOBOT/research/ APweb/). Acceso: 02/11/2012.

Stohlgren, T.; Falkner, M.; Schell, L. 1995. A Modified-Whittaker Nested Vegetation Sampling Method. Vegetatio, 117 (2): 113-121.

Terborgh, J.; Andresen, E. 1998. The composition of Amazonian forests: patterns at local and regional scales. Journal of Tropical Ecology, 14: 645-664.

Ter Steege, H.; Pitman, N.; Sabatier, D.; Castellanos, H.; van der Hout, P.; Douglas, D. C.; Silveira, M.; Phillips, O.; Vásquez, R.; van Andel, T.; Duivenvoorden, J.; Adalardo de Oliveira, A.; Ek, R.; Lilwah, R.; Thomas, R.; van Essen, J.; Baider, C.; Maas, P.; Mori, S.; Terborgh, J.; Nuñez, P.; Mogollón, H. \& W. Morawetz. 2003. A spatial model of tree $\alpha$ diversity and tree density for the Amazon. Biodiversity and Conservation 12: 2255-2277.

Valderrama, E. 2007. Estudio florístico en una hectárea de bosque colinoso en la estación experimental del Instituto Tecnológico de 
Nauta, Loreto-Perú. Tesis de pre-grado, Universidad Nacional de la Amazonía Peruana, Facultad de Ciencias Biológicas, Iquitos, departamento de Loreto, Perú. 45pp.

Vásquez, R. 1997. Flórula de las Reservas Biológicas de Iquitos, Perú. Missouri Botanical Garden Press. St. Louis-USA. 1046pp.

Vásquez, R.; Rojas, R. 2005. FLORA DEL RÍO CENEPA, AMAZONAS - PERÚ, Introducción a la Diversidad Florística de un Refugio de Endemismos y Disyunciones. (http://www.geocities.com/jbmperu/condor.ht m). Acceso: 15/04/2010.

Vásquez, R.; Rojas, R.; Rodríguez, E. 2002. Adiciones a la Flora Peruana: especies nuevas, nuevos registros y estados taxonómicos de las Angiospermas para el Perú. Arnaldoa 9 (2): 43110.
Vásquez, R.; R. Rojas. 2004. Plantas de la Amazonía Peruana Clave para Identificar las Familias de Gymnospermae y Angiospermae. Arnaldoa 261 págs.

Vriesendorp, C.; Pitman, N.; Foster, R.; Mesones, I.; Rios, M. 2004. Flora y Vegetación. In: Pitman, N.; Smith, R.; Vriesendorp, C.; Moskovits, D.; Piana, R.; Knell, G.; Wachter, T. (eds). Peru: Ampiyacu, Apayacu, Yaguas, Medio Putumayo. Rapid Biological Inventories Report 12. p. 5461.

Wittmann, F.; Schöngart, J.; Montero, J.; Motzer, T.; Junk, W.; Piedade, M.; Queiroz, H.; Worbes, M. 2006. Tree species composition and diversity gradients in white-water forests across the Amazon Basin. Journal of Biogeography 33, 1334-1347p.

Warburg, O. 1897. Monographie der Myristicaceae. Nova Acta Acad. Caes. Leop.-Carol. 68: 1-680.

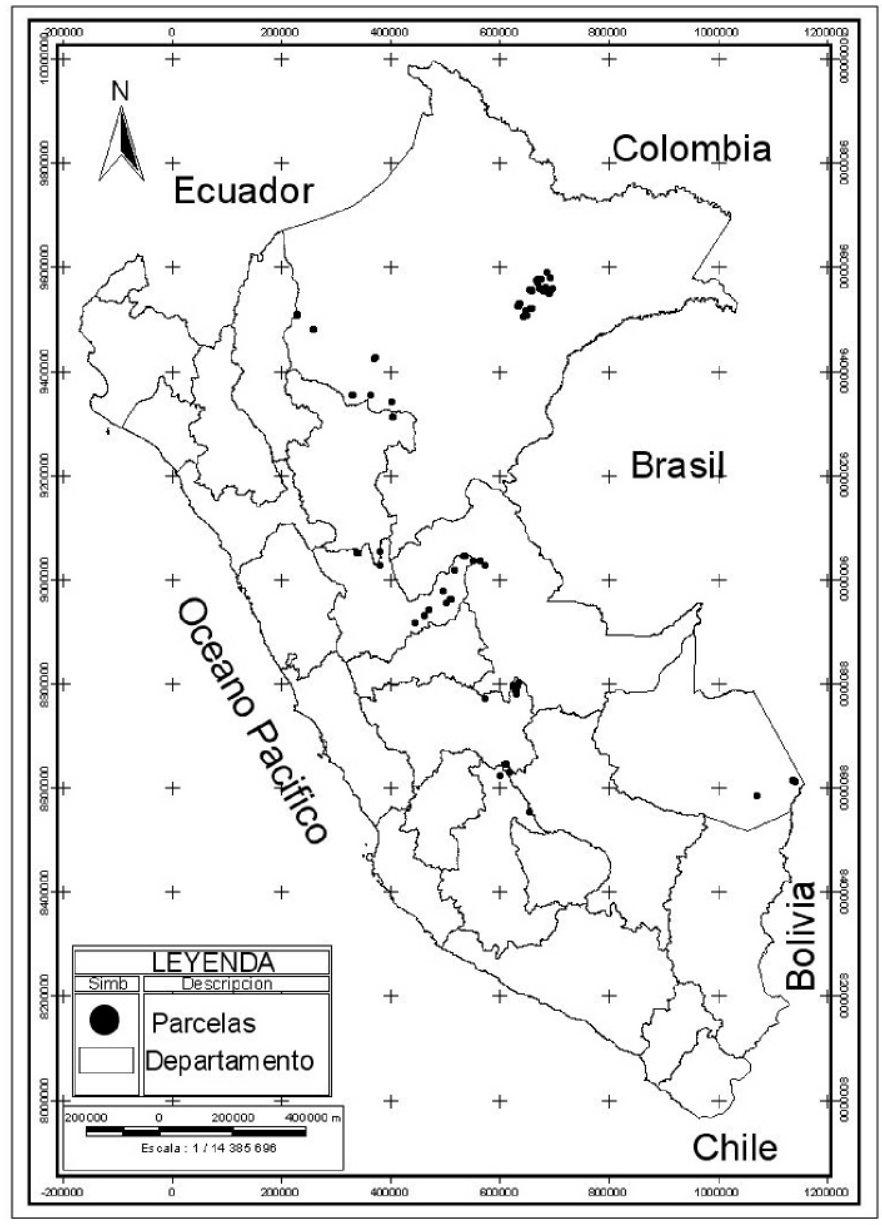

Figura 1. Ubicación de las 100 parcelas de muestreo en la Amazonía peruana. 


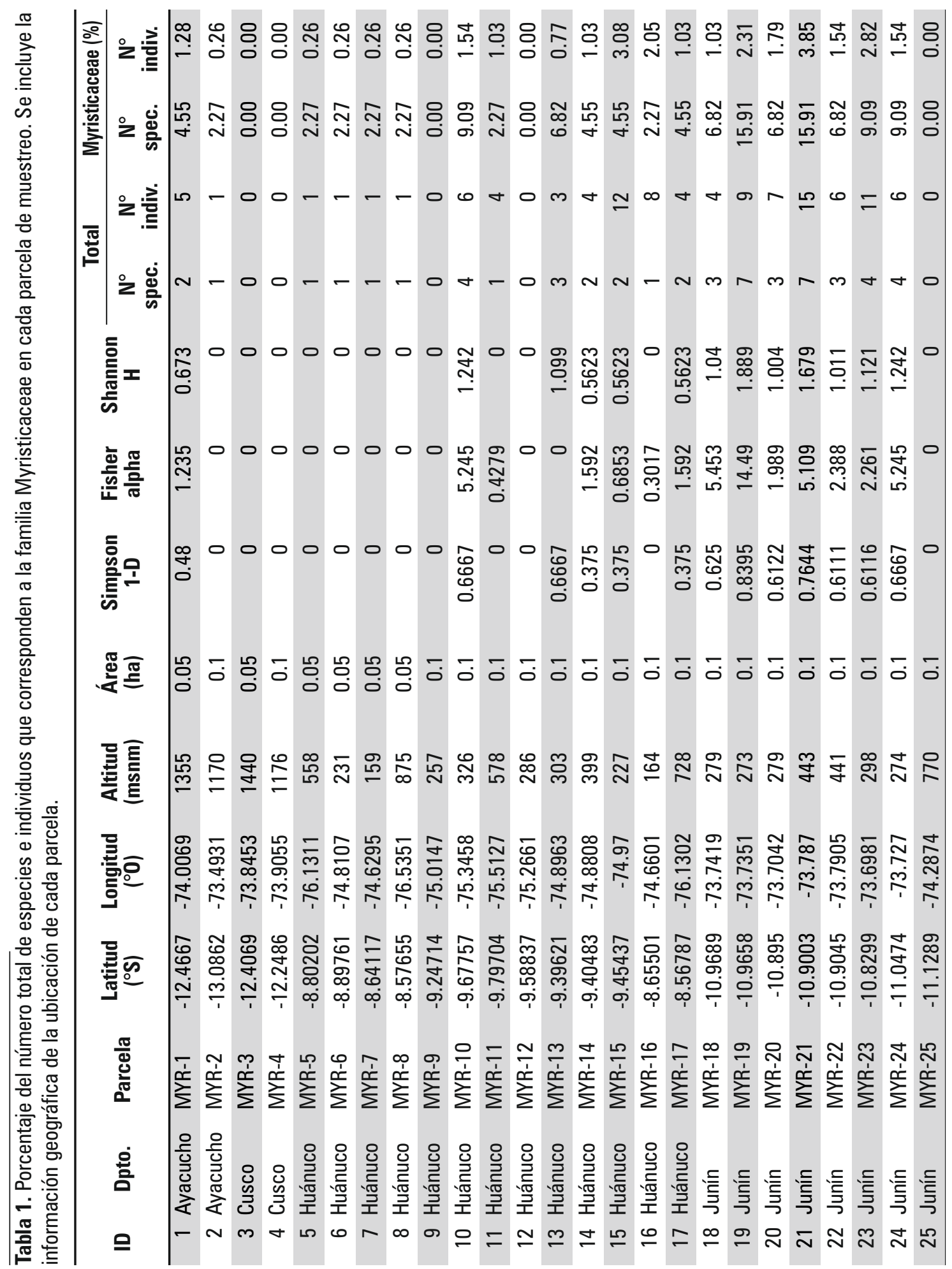




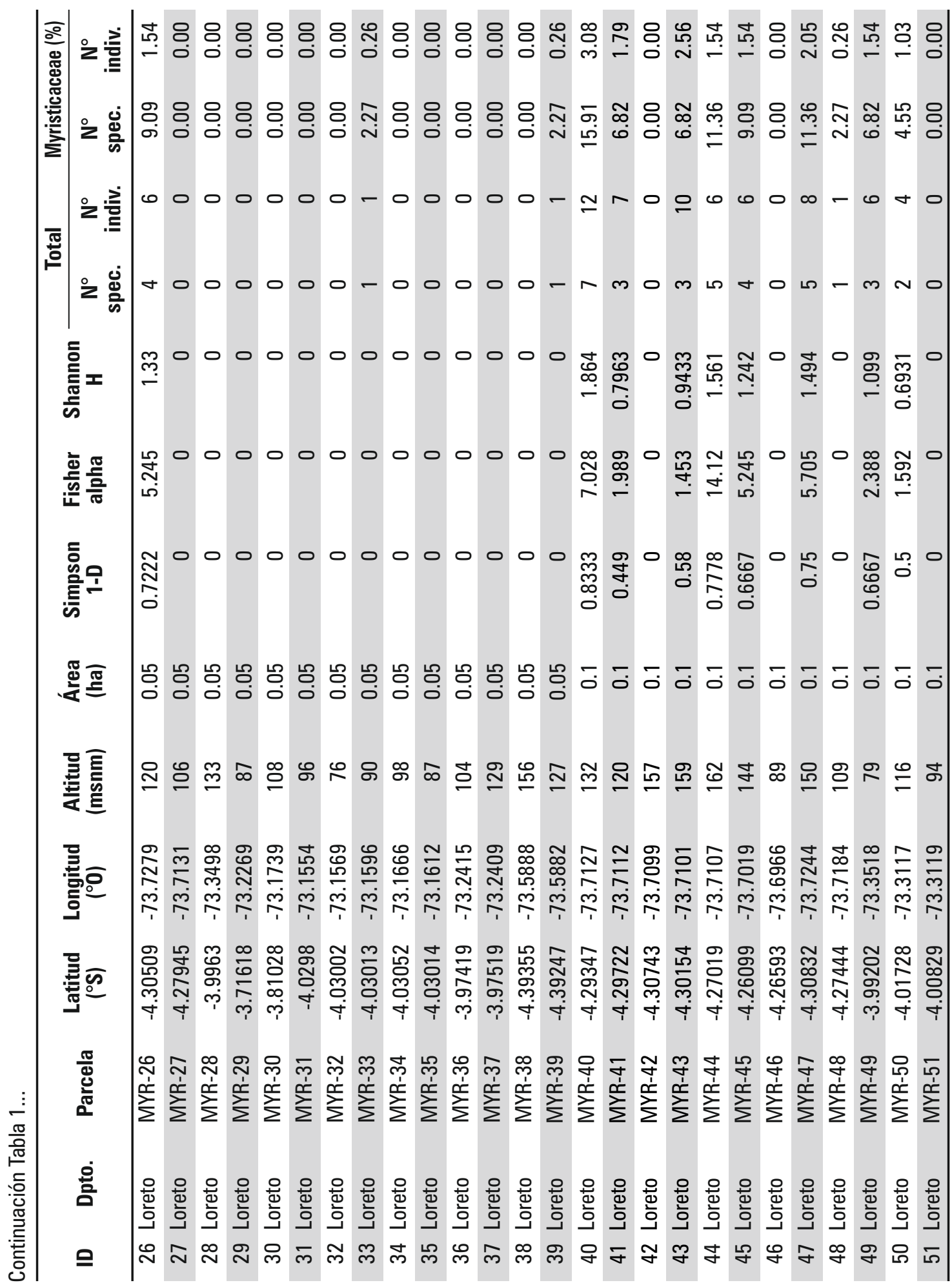


FOLIA $\mid$ INVENTARIO DE CUMALAS (MYristicaCEAE)

Amazónica EN LA AMAZONí́ PERUANA

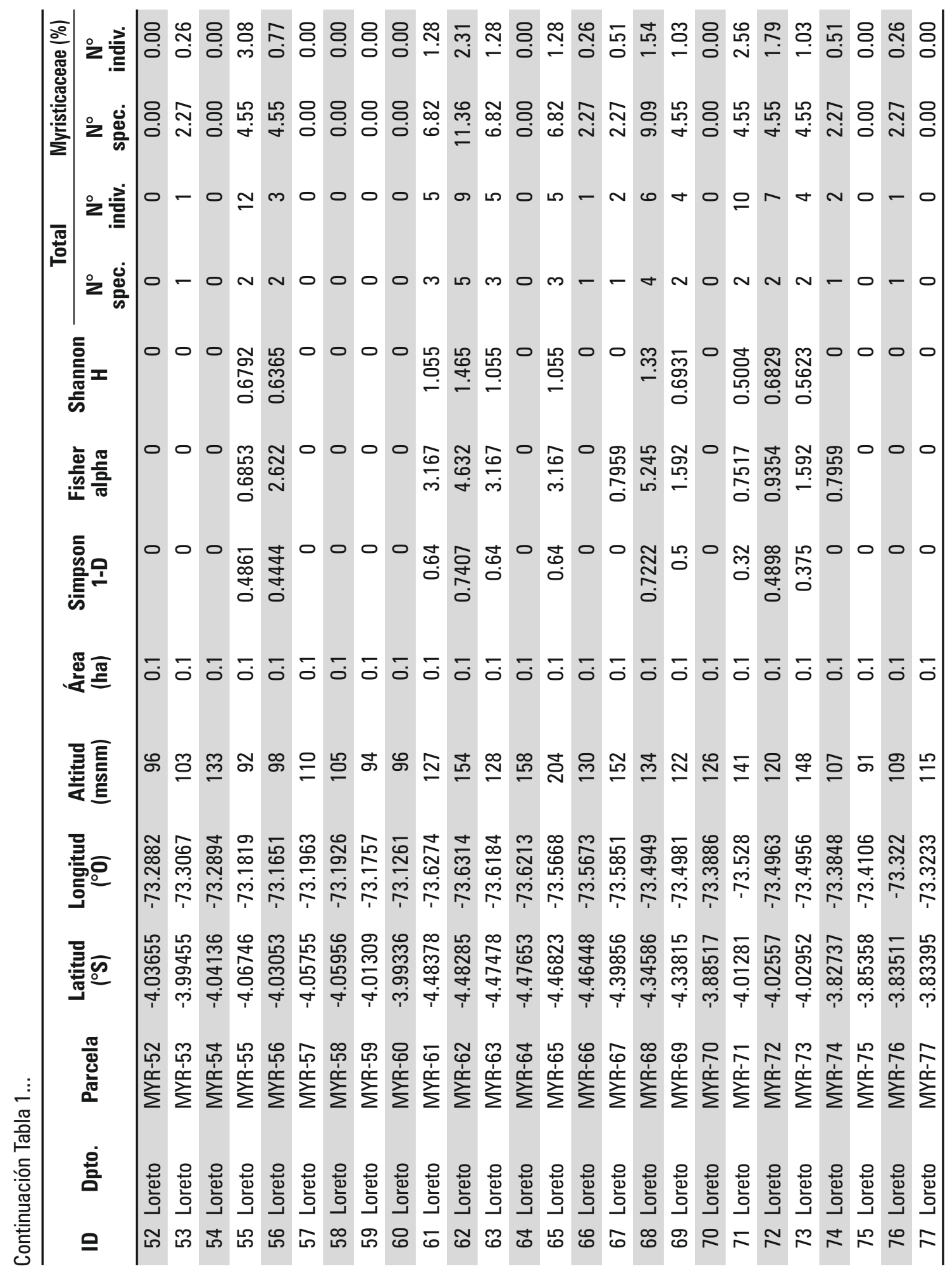




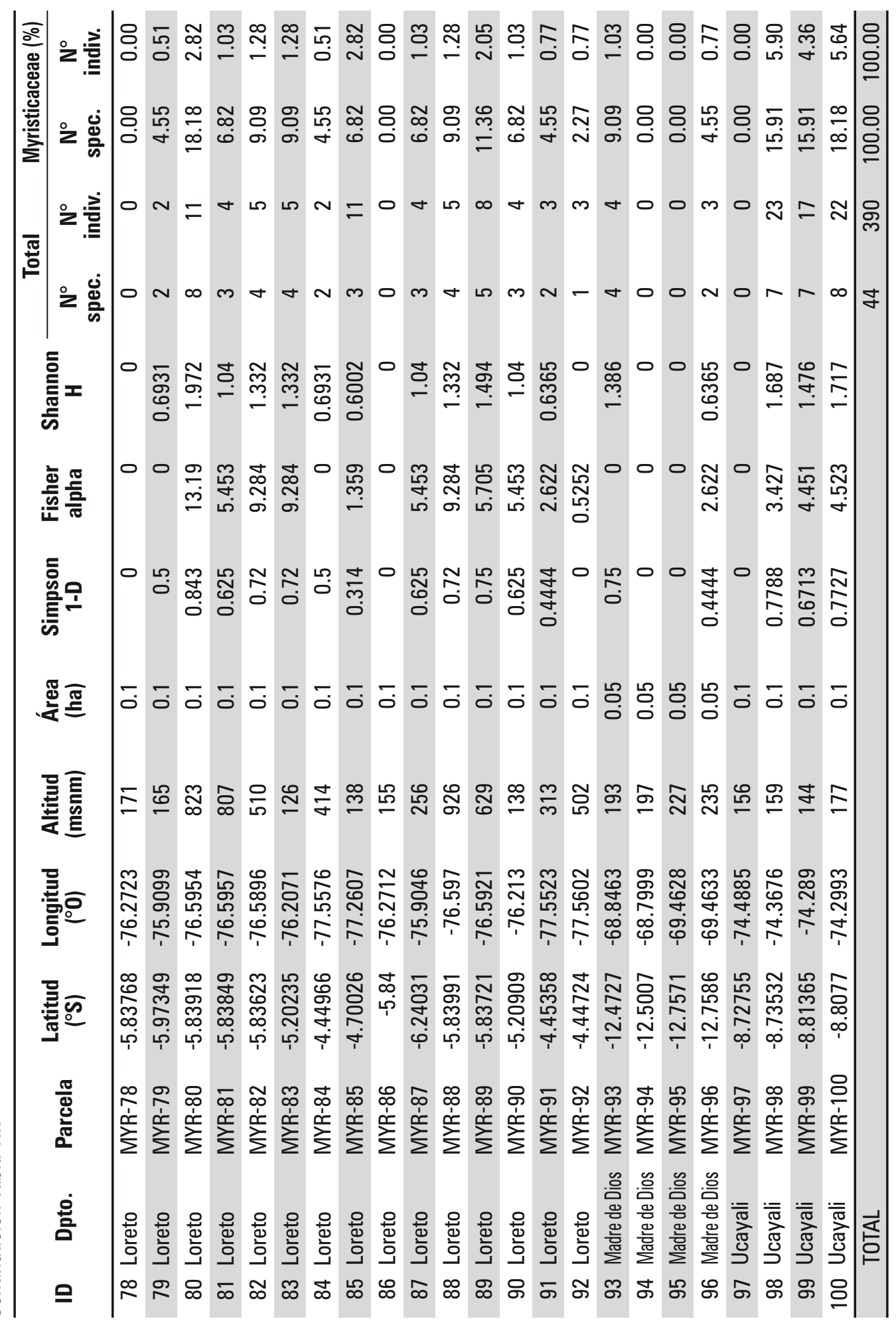


FOLIA

Tabla 2. Especies con cantidad de individuos reportadas para la Amazonía peruana de acuerdo a este estudio.

\begin{tabular}{|c|c|c|c|c|c|c|c|}
\hline Id & Especie & Ind. & $\%$ & Id & Especie & Ind. & $\%$ \\
\hline 1 & Virola pavonis & 82 & 21.1 & 23 & Virola loretensis & 3 & 0.8 \\
\hline 2 & Iryanthera laevis & 43 & 11 & 24 & Virola sp. 2 & 3 & 0.8 \\
\hline 3 & Otoba glycycarpa & 35 & 9 & 25 & Compsoneura sprucei & 2 & 0.5 \\
\hline 4 & Virola calophylla & 28 & 7.2 & 26 & Iryanthera sp. 3 & 2 & 0.5 \\
\hline 5 & Otoba parvifolia & 19 & 4.9 & 27 & Iryanthera sp. 5 & 2 & 0.5 \\
\hline 6 & Iryanthera juruensis & 17 & 4.4 & 28 & Virola duckei & 2 & 0.5 \\
\hline 7 & Iryanthera paraensis & 17 & 4.4 & 29 & Virola multinervia & 2 & 0.5 \\
\hline 8 & Iryanthera tricornis & 16 & 4.1 & 30 & Virola obovata & 2 & 0.5 \\
\hline 9 & Iryanthera hostmannii & 14 & 3.6 & 31 & Virola sebifera & 2 & 0.5 \\
\hline 10 & Iryanthera tessmannii & 13 & 3.3 & 32 & Virola sp. 3 & 2 & 0.5 \\
\hline 11 & Virola elongata & 12 & 3.1 & 33 & Virola sp. 5 & 2 & 0.5 \\
\hline 12 & Iryanthera lancifolia & 8 & 2.1 & 34 & Virola surinamensis & 2 & 0.5 \\
\hline 13 & Osteophloeum platyspermum & 7 & 1.8 & 35 & Iryanthera olacoides & 1 & 0.3 \\
\hline 14 & Compsoneura capitellata & 6 & 1.5 & 36 & Iryanthera sp. 1 & 1 & 0.3 \\
\hline 15 & Iryanthera crassifolia & 6 & 1.5 & 37 & Iryanthera sp. 2 & 1 & 0.3 \\
\hline 16 & Iryanthera elliptica & 6 & 1.5 & 38 & Virola albidiflora & 1 & 0.3 \\
\hline 17 & Iryanthera sp. 4 & 5 & 1.3 & 39 & Virola divergens & 1 & 0.3 \\
\hline 18 & Virola peruviana & 5 & 1.3 & 40 & Virola duckei cf. & 1 & 0.3 \\
\hline 19 & Virola sp. 1 & 5 & 1.3 & 41 & Virola mollissima & 1 & 0.3 \\
\hline 20 & Virola flexuosa & 4 & 1 & 42 & Virola multiflora & 1 & 0.3 \\
\hline 21 & Iryanthera macrophylla & 3 & 0.8 & 43 & Virola sebifera cf. & 1 & 0.3 \\
\hline 22 & Virola decorticans & 3 & 0.8 & 44 & Virola sp. 4 & 1 & 0.3 \\
\hline \multicolumn{8}{|c|}{ Total $=390$} \\
\hline
\end{tabular}

Tabla 3. Géneros con cantidad de especies e individuos reportadas para la Amazonía peruana de acuerdo a este estudio.

\begin{tabular}{lrrrr}
\hline \multicolumn{1}{r}{ Género } & Especies & $\%$ & Individuos & $\%$ \\
\hline Virola & 23 & 52.3 & 166 & 42.6 \\
Iryanthera & 16 & 36.4 & 155 & 39.7 \\
Compsoneura & 2 & 4.5 & 54 & 13.8 \\
Otoba & 2 & 4.5 & 8 & 2.1 \\
Osteophloeum & 1 & 2.3 & 7 & 1.8 \\
\hline Total general & $\mathbf{4 4}$ & $\mathbf{1 0 0}$ & $\mathbf{3 9 0}$ & $\mathbf{1 0 0}$ \\
\hline
\end{tabular}




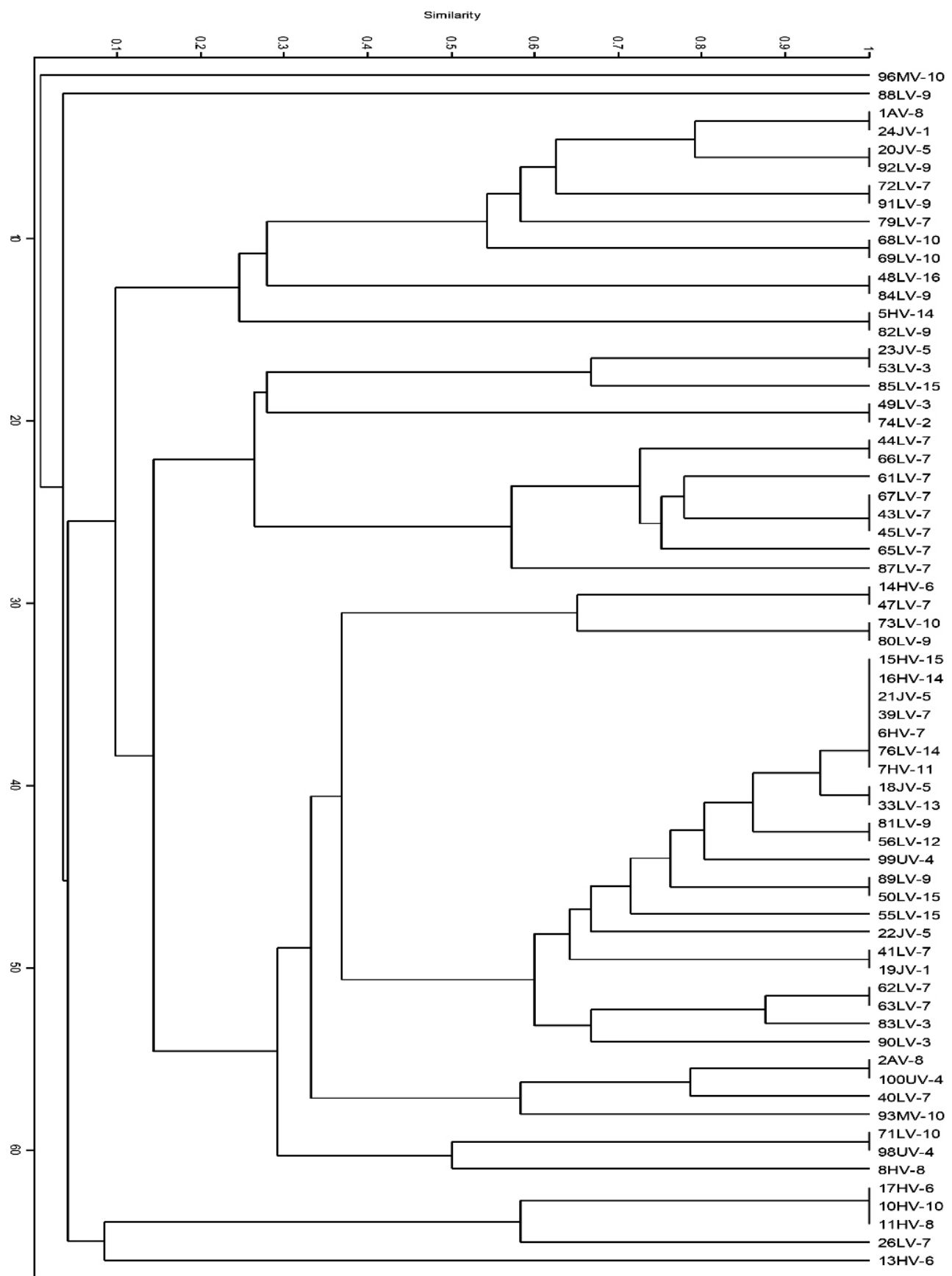

Leyenda: Bosques con Bambu (V-1), Bosques inundable por aguas negras (V-2), Bosques Pantanoso de especies heterogéneas (V-3), Bosques de planicies del suroeste de la Amazonia (V-4), Bosques subandino del suroeste de la Amazonia (V-5), Bosques de colinas altas (V-6), Bosques de colinas bajas (V-7), Bosques de montañas altas (V-8), Bosques de Montañas bajas (V-9), Bosques de planicies no inundables del oeste de la Amazonía (V-10), Bosques inundables por aguas blancas (V-11), Complejo sucesional ripario diverso (V-12), Comunidad de Montrichardia arborea (V13), Palmeral denso (aguajal denso) (V-14), Palmeral mixto (aguajal mixto) (V-15), Vegetación riparia (V-16).

Figura 2. Dendrograma del índice de similitud de Simpson de la composición florística de Myristicaceae entre las parcelas establecidas. 


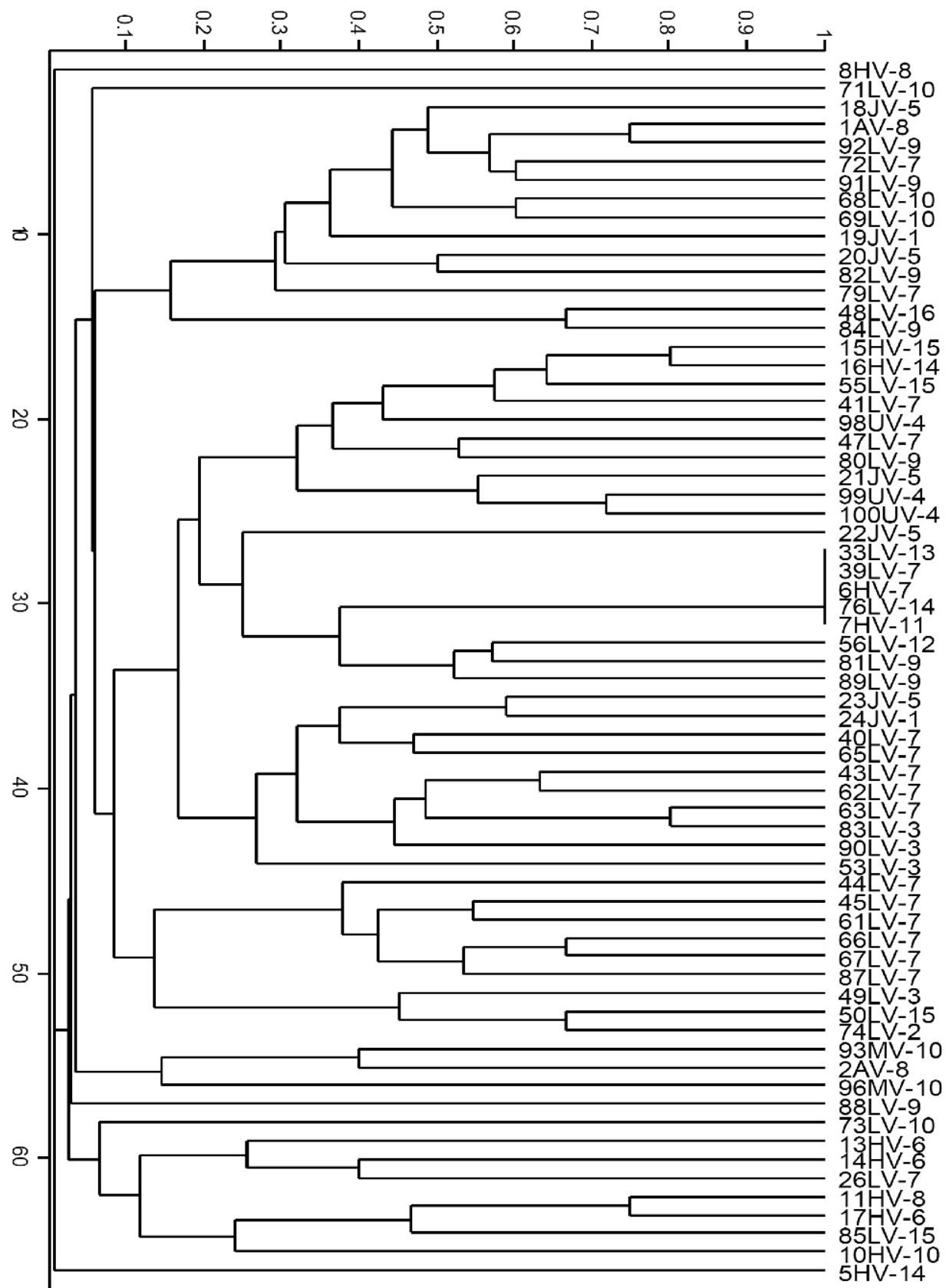

Leyenda: Bosques con Bambu (V-1), Bosques inundable por aguas negras (V-2), Bosques Pantanoso de especies heterogéneas (V-3), Bosques de planicies del suroeste de la Amazonia (V-4), Bosques subandino del suroeste de la Amazonia (V-5), Bosques de colinas altas (V-6), Bosques de colinas bajas (V-7), Bosques de montañas altas (V-8), Bosques de Montañas bajas (V-9), Bosques de planicies no inundables del oeste de la Amazonía (V-10), Bosques inundables por aguas blancas (V-11), Complejo sucesional ripario diverso (V-12), Comunidad de Montrichardia arborea (V13), Palmeral denso (aguajal denso) (V-14), Palmeral mixto (aguajal mixto) (V-15), Vegetación riparia (V-16).

Figura 3. Dendrograma del índice de similitud de Bray-Curtis de la composición florística de Myristicaceae entre las parcelas establecidas. 

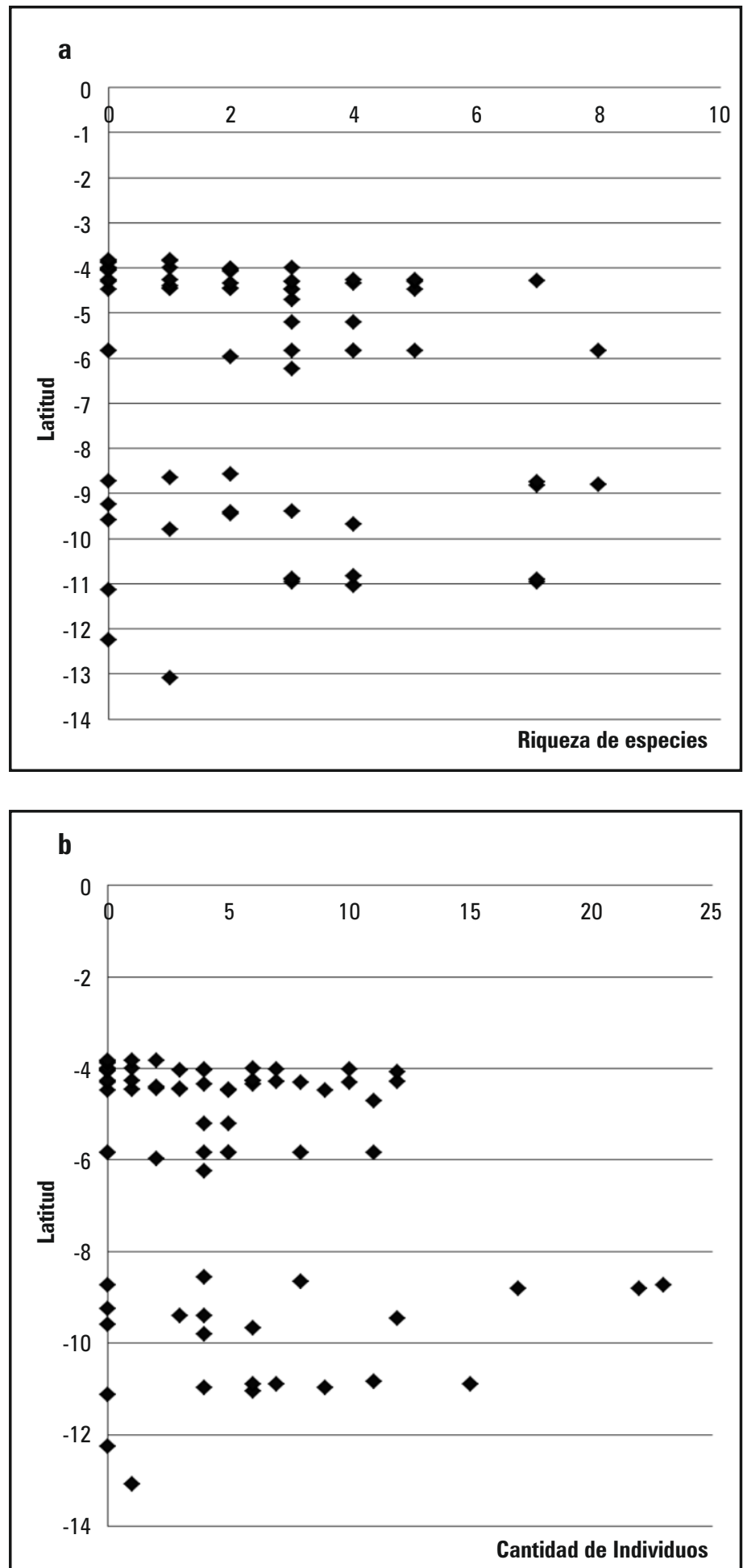

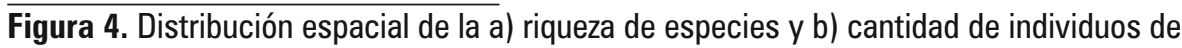
Myristicaceae según gradiente latitudinal en la Amazonía peruana. 


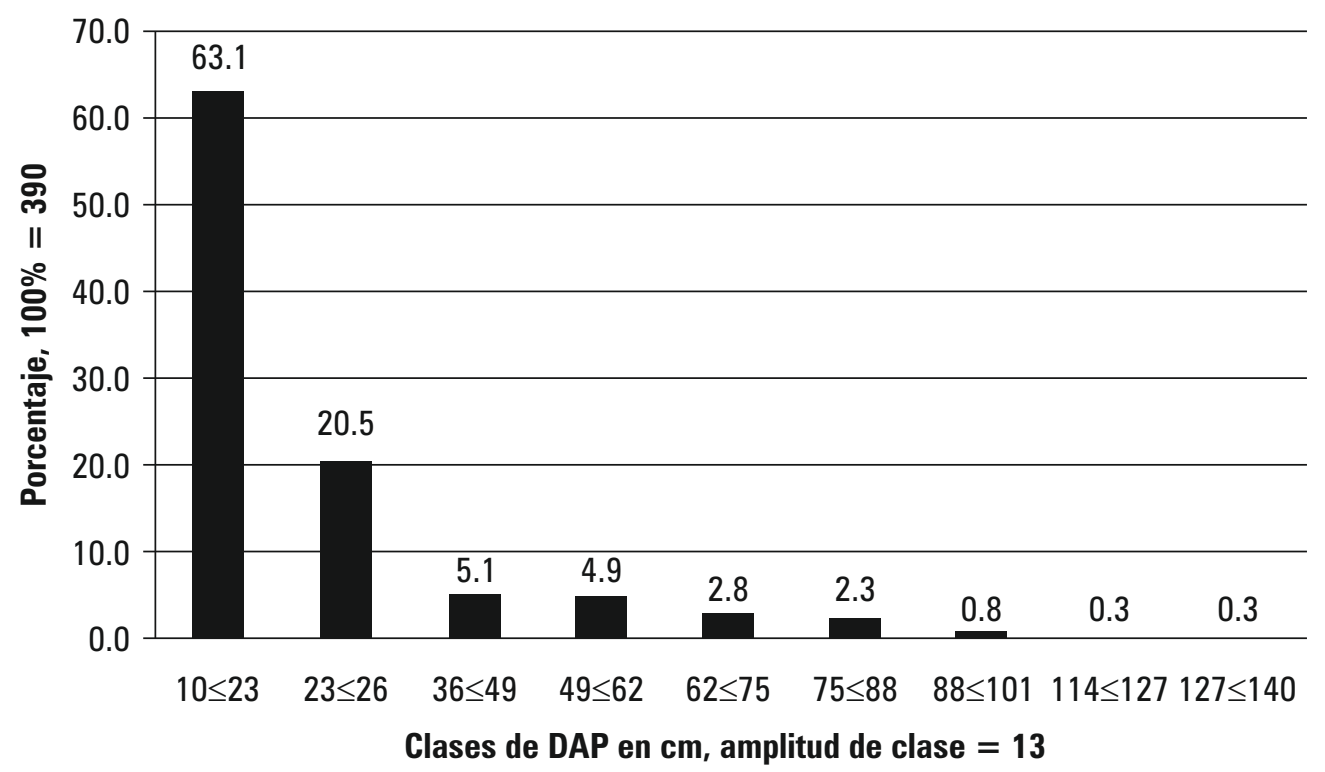

Figura 4. Distribución de clases diamétricas según el número de individuos de Myristicaceae $\left(n_{\text {total }}=390\right.$ individuos) reportadas en 100 parcelas de muestreo.

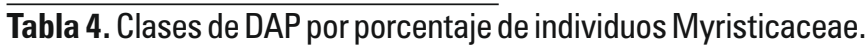

\begin{tabular}{lcc}
\hline \multicolumn{1}{c}{ Clase de DAP $(\mathbf{c m})$} & Individuos & $\%$ \\
\hline $10 \leq 23$ & 246 & 63.1 \\
$23 \leq 36$ & 80 & 20.5 \\
$36 \leq 49$ & 20 & 5.1 \\
$49 \leq 62$ & 19 & 4.9 \\
$62 \leq 75$ & 11 & 2.8 \\
$75 \leq 88$ & 9 & 2.3 \\
$88 \leq 101$ & 3 & 0.8 \\
$114 \leq 127$ & 1 & 0.3 \\
\hline $127 \leq 140$ & 1 & 0.3 \\
\hline
\end{tabular}

